\title{
Variación intra-específica en Nothofagus glauca una especie endémica de los bosques mediterráneos de Chile
}

\author{
Intra-specific variation in Nothofagus glauca, an endemic species \\ of the Mediterranean forests of Chile
}

\author{
Rómulo Santelices-Moya ${ }^{a *}$, Rodrigo Vergara ${ }^{b}$, Antonio Cabrera-Ariza ${ }^{c}$, \\ Sergio Espinoza-Meza ${ }^{\text {d, }}$ Patricia Silva-Flores ${ }^{\text {e }}$

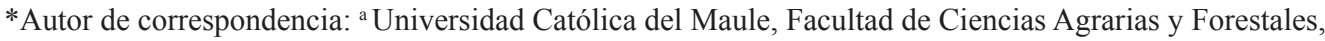 \\ Centro del Secano, Talca, Chile, tel.: 56-71-2203501,rsanteli@ucm.cl \\ ${ }^{\mathrm{b}}$ Consultor independiente, Talca, Chile. \\ c Universidad Católica del Maule, Centro de Investigación de Estudios Avanzados del Maule (CIEAM) y Centro del Secano, \\ Talca, Chile. \\ ${ }^{\mathrm{d}}$ Universidad Católica del Maule, Facultad de Ciencias Agrarias y Forestales, Depto. de Ciencias Forestales, Talca, Chile. \\ ${ }^{e}$ Universidad Católica del Maule, Centro de Investigación de Estudios Avanzados del Maule (CIEAM), \\ Vicerrectoría de Investigación y Posgrado, y Centro del Secano, Talca, Chile.
}

\begin{abstract}
SUMMARY
In forest conservation, especially in a climate change scenario, to guide decision-making, it is essential to know the intra-specific variation of multiple traits that exist in a species. Therefore, the objective of this work was to analyze the current state of knowledge about the variation of Nothofagus glauca. In this way, guidelines may be proposed for future programs for the conservation of genetic resources or the restoration of forests of this species. Important intra-specific variability is analyzed and evidenced in aspects related to its spatial distribution, its variation in ecological distribution, its populations associated with the forest typology, its genecological differentiation, its variation in growth and its genetic variation detected by isoenzymatic and molecular markers. Based on the abundant research, a step to a second experimental phase is proposed to select populations capable of adapting to the new environmental conditions resulting from climate change, which must consider the microbiome of this species. It is proposed that, in this way, efficient conservation and restoration of $N$. glauca will be achieved.
\end{abstract}

Key words: hualo, ecological variation, genecology, growth, genetic variation.

\section{RESUMEN}

En el contexto de la conservación de bosques, especialmente en un escenario de cambio climático, es fundamental conocer la variación intra-específica de múltiples rasgos que existe en una especie, a fin de orientar la toma de decisiones. Por ello, el objetivo de este trabajo fue analizar el estado actual del conocimiento sobre la variación de Nothofagus glauca de tal forma de proponer lineamientos para futuros programas de conservación de los recursos genéticos o la restauración de los bosques de esta especie. Se analizan y evidencia gran variabilidad intra-específica en aspectos relacionados con su distribución espacial; su variación en la distribución ecológica; sus poblaciones asociadas a la tipología forestal; su diferenciación genecológica; su variación en crecimiento; y su variación genética detectada mediante marcadores isoenzimáticos y moleculares. A base de la abundante investigación existente se propone un salto a una segunda fase experimental para seleccionar poblaciones capaces de adaptarse a las nuevas condiciones ambientales producto del cambio climático, las cuales deben considerar el microbioma de esta especie. Se propone que de esta forma se logrará una eficiente conservación y restauración de $N$. glauca.

Palabras clave: hualo, variación ecológica, genecología, crecimiento, variación genética.

\section{INTRODUCCIÓN}

Nothofagus glauca (Phil.) Krasser (hualo, roble maulino o roble colorado), es una especie endémica de la zona mediterránea de Chile perteneciente a la familia Notho- fagaceae (Santelices et al. 2013a). Nothofagus glauca es un árbol monoico caducifolio, que puede llegar a medir hasta $30 \mathrm{~m}$ de altura. Se ha descrito que tiene un fuste cilíndrico de hasta $2 \mathrm{~m}$ de diámetro (Rodríguez y Quezada 2003), pero en la actualidad es difícil encontrar ejempla- 
res que tengan más de $40 \mathrm{~cm}$ de diámetro. Es una especie que se encuentra catalogada como vulnerable a la extinción (Barstow et al. 2017), aunque el Estado de Chile la clasifica como especie fuera de peligro según el Decreto Supremo 51/2008 del Ministerio Secretaría General de la Presidencia (MSEGPRES 2008).

Los bosques de $N$. glauca se insertan en la formación conocida como Bosque Maulino, que es un sistema de transición entre las formaciones xerofíticas y los bosques templados más meridionales. En esta zona, los bosques caducifolios están adaptados a los períodos secos prolongados del verano y cumplen un rol muy importante en la conservación del agua y el suelo orgánico, en el ciclo biogeoquímico del carbono, y ofrecen una gran variedad de nichos ecológicos y hábitats a la flora, fauna y microbiota asociada (Arroyo et al. 1996). Aquí, además, se concentra la mayor densidad poblacional de Chile y, en consecuencia, existe una fuerte presión antropogénica sobre los recursos naturales, que ha producido un fuerte retroceso de los bosques de esta región.

Los sistemas mediterráneos por sus características son más propensos a los incendios forestales y, como consecuencia del cambio climático (i.e. aumento de la temperatura y baja humedad), sumado a fuertes vientos, se favorecerán condiciones como las que ya dieron origen en esta zona a uno de los mayores incendios en el año 2017, afectando 184.000 ha de sistemas agrícolas y forestales (Valencia et al. 2018), incluyendo parte importante de los bosques remanentes de $N$. glauca.

Actualmente, ante la amenaza que significa el cambio climático, es fundamental conocer la variación que existe en una especie, a fin de orientar la toma de decisiones, en especial en orden a la conservación de los recursos genéticos. Para muchas especies arbóreas de los bosques nativos de Chile se ha descrito la variación intra-específica, incluyendo la mayoría del género Nothofagus. No obstante, aún está pendiente la descripción de la variación para las especies de Nothofagus de la zona mediterránea. Por ello, el objetivo de este trabajo es analizar el estado actual del conocimiento sobre la variación de $N$. glauca de tal forma de proponer lineamientos para futuros programas de conservación de los recursos genéticos y la restauración de los bosques de esta especie, la más representativa de los bosques mediterráneos del género Nothofagus de Sudamérica.

\section{DISTRIBUCIÓN GENERAL}

Considerando la presencia de la especie en forma mayoritaria en el dosel superior y siguiendo la información entregada por Le-Quesne y Sandoval (2001) y la Corporación Nacional Forestal (SINIA 2019), tras un análisis con herramientas de SIG se estima que la superficie cubierta por los bosques de $N$. glauca en su distribución natural es de 156.972 ha, distribuyéndose un $29 \%$ en la Cordillera de la Costa y un $71 \%$ en la Cordillera de Los Andes (cuadro 1). Comprende parte de las altitudes medias de la Cordillera de la Costa y de la Cordillera de Los Andes (figura 1). Geo- gráficamente se distribuye en forma intermitente desde la Región Metropolitana de Santiago, hasta la Región del Biobío. La formación más septentrional aparece en la comuna de Alhué, provincia de Melipilla (33⒌ $58^{\prime} \mathrm{S}-71^{\circ} 05^{\prime} \mathrm{W}$, $614 \mathrm{~m}$ s.n.m.), mientras que la más meridional, de acuerdo a Le-Quesne y Sandoval (2001), corresponde a un rodal en la comuna de Quilleco en la provincia de Biobío ( $37^{\circ} 27^{\prime} \mathrm{S}$ $71^{\circ} 58^{\prime}$ W, 340 m s.n.m.), a unos $100 \mathrm{~km}$ al sur de la distribución original en la provincia de Punilla. En la Región del Maule se concentra la mayor superficie de bosques de $N$. glauca (146.770 ha, $94 \%$ del recurso), principalmente en la Cordillera de Los Andes en las comunas de Colbún, Linares, y Parral. En la Cordillera de Los Andes, N. glauca aparece en forma discontinua en los $34^{\circ} 33^{\prime}$ de latitud Sur, sobre los $1.000 \mathrm{~m}$ de altitud, donde tiende a formar bosques puros. Entre los ríos Maule y Nuble se concentra la mayor abundancia de los bosques andinos de $N$. glauca (casi un $64 \%$ ). En la Cordillera de la Costa, $N$. glauca ha decrecido fuertemente producto de talas indiscriminadas de sus bosques y del reemplazo por otras especies de rápido crecimiento. Las poblaciones más septentrionales aparecen en la Región Metropolitana de Santiago, teniendo presencia en forma discontinua hasta la Región del Ñuble. La mayor concentración se localiza en la Región del Maule (23 \%).

\section{VARIACIÓN EN LA DISTRIBUCIÓN ECOLÓGICA DE NOTHOFAGUS GLAUCA}

El clima de la zona donde se distribuye $N$. glauca es del tipo mediterráneo, con estaciones bien marcadas, con una concentración de las precipitaciones en el invierno y con un periodo estival seco que, en algunos lugares, puede llegar incluso a presentar cinco meses con ausencia total de

Cuadro 1. Distribución regional y provincial, de los bosques de Nothofagus glauca (Le-Quesne y Sandoval 2001, SINIA 2019).

Regional and provincial distribution of Nothofagus glauca forests (Le-Quesne and Sandoval 2001, SINIA 2019).

\begin{tabular}{llcc}
\hline \multirow{2}{*}{ Región } & \multirow{2}{*}{ Provincia } & \multicolumn{2}{c}{ Superficie } \\
\cline { 3 - 4 } Metropolitana & Melipilla & (ha) & $(\%)$ \\
\hline O’Higgins & Cachapoal & $1.837,5$ & 0,01 \\
& Colchagua & $1.766,3$ & 1,17 \\
\hline Maule & Curicó & $4.356,3$ & 2,78 \\
& Talca & $33.943,5$ & 21,62 \\
& Linares & $95.793,9$ & 61,03 \\
& Cauquenes & $12.676,6$ & 8,08 \\
\hline Ñuble & Punilla & $6.577,0$ & 4,18 \\
\hline Biobío & Biobío & 1,0 & 0,0 \\
\hline Total & & $156.972,2$ & 100,0 \\
\hline
\end{tabular}




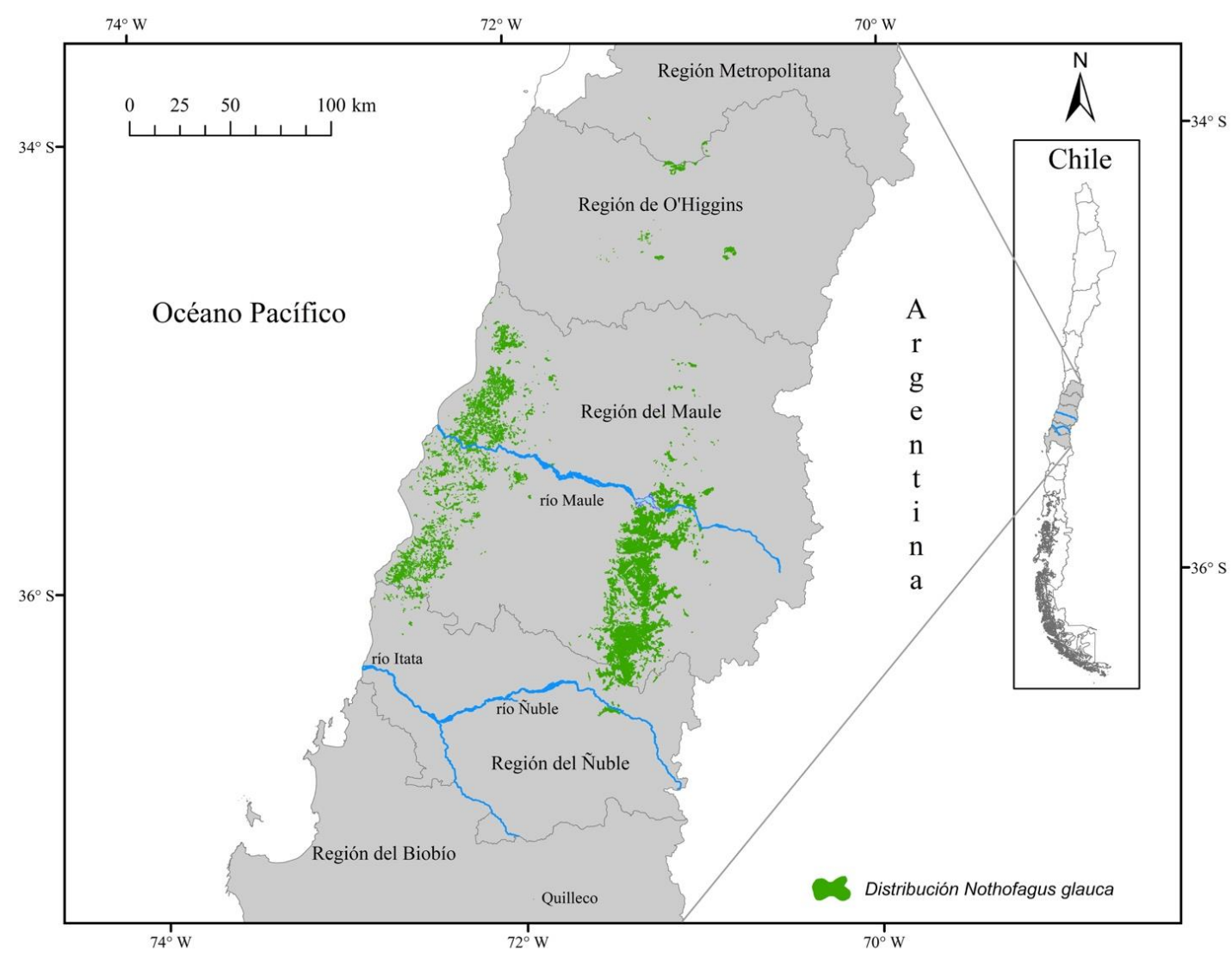

Figura 1. Distribución espacial de los bosques de Nothofagus glauca (Le-Quesne y Sandoval 2001, SINIA 2019).

Spatial distribution of Nothofagus glauca forests (Le-Quesne and Sandoval 2001, SINIA 2019).

precipitaciones. Según la clasificación de Köppen (Rioseco y Tesser 2016), corresponde a un clima de latitudes medias, en el que se pueden distinguir tres zonas climáticas: la Csbn's es la distribución que tiene más influencia del mar como efecto termorregulador, con un clima templado con lluvias invernales y elevada humedad atmosférica; la Csb, tanto en la Cordillera de la Costa como en la Cordillera de Los Andes, con un clima templado cálido con lluvias invernales; y la Csc, en la distribución andina más oriental, con un clima templado frío con lluvias invernales. En el área costera se presenta un clima templado-cálido con estación seca prolongada e influencia marítima, con una alta humedad relativa. La temperatura media anual, entre los 300 y $553 \mathrm{~m}$ de altitud, varía entre 12,3 y $13,1^{\circ} \mathrm{C}$, mientras que las medias extremas son $6,1^{\circ} \mathrm{C}$ la mínima y $22,9^{\circ} \mathrm{C}$ la máxima. La precipitación media anual oscila entre 844 y $1.296 \mathrm{~mm}$ (López 1994), aunque en la actualidad y como consecuenia del cambio climático las precipitaciones han disminuido. En la región andina, el clima es templado-cálido con estación seca de cuatro a cinco meses. Entre los 300 y $450 \mathrm{~m}$ s.n.m., la temperatura media anual varía de 12,5 a $13,9{ }^{\circ} \mathrm{C}$ y las medias extremas son $4,4{ }^{\circ} \mathrm{C}$ la mínima y $28,2{ }^{\circ} \mathrm{C}$ la máxima. La precipitación media anual se presenta en un rango entre 1.134 y $1.570 \mathrm{~mm}$ (López 1994), aunque al igual que en la Cordillera de la Costa, las precipitaciones en la actualidad han disminuido por efecto del cambio climático. En las partes de mayor altitud de la distribución andina, la precipitación se puede presentar en forma de nieve.

Tal como sucede con otras especies del Bosque Maulino, los bosques de $N$. glauca han estado sujetos a una fuerte presión antropogénica, resultando de ello su fragmentación. Especialmente en la Cordillera de la Costa, los bosques remanentes se encuentran dentro de una matriz boscosa compuesta por especies alóctonas de rápido crecimiento, en donde destaca la presencia de Pinus radiata D. Don (Santelices et al. 2013a). Sin la presencia de un disturbio, $P$. radiata no es capaz de colonizar un bosque fragmentado de $N$. glauca, a pesar de que sus semillas sean dispersadas al interior del bosque nativo (Bustamante y Simonetti 2005). Sin embargo, en forma posterior a un disturbio de la magnitud de un incendio forestal, $P$. radiata invade con éxito los bosques de $N$. glauca. Por ello, y gracias a sus características biológicas y a su vasta presencia, $P$. radiata tiene la capacidad de establecerse y dominar el sitio, lo que puede provocar cambios del ecosistema afectando, por ejemplo, el ciclo de nutrientes y el ciclo del agua (Litton y Santelices 2003), convirtiéndose en definitiva en una amenaza para estos bosques. La presencia del fuego en la zona 
mediterránea de Chile, donde se distribuye naturalmente $N$. glauca y se concentra la mayor densidad poblacional de Chile, se ha visto favorecida últimamente por la presencia del fenómeno de El Niño (mayor temperatura y aumento de combustible). En los últimos cinco años se ha registrado una persistente sequía en esta zona, con precipitaciones incluso menores a un $70 \%$ respecto a un año normal. También se ha observado un leve aumento en las temperaturas.

En el área de distribución de $N$. glauca se encuentran diversas variaciones climáticas. A nivel longitudinal se distinguen aquellas formaciones localizadas en la Cordillera de la Costa y en la Cordillera de Los Andes. Más marcada es la variación climática a lo largo de la gradiente latitudinal, teniendo los bosques más septentrionales, como es lógico, condiciones de mayor aridez que aquellos más meridionales. Por ejemplo, en su distribución más septentrional (comunas de Alhué y San Pedro), la precipitación media anual varía entre 537 y $676 \mathrm{~mm}$; por el contrario, en la distribución andina en las inmediaciones del río Ñuble, la precipitación media anual se presenta en un rango entre 1.247 y $1.348 \mathrm{~mm}$ anuales; en la Cordillera de la Costa, en su distribución más meridional, este rango va de 908 a $984 \mathrm{~mm}$ anuales (Hijmans et al. 2005). Ello podría ser un factor de diferenciación entre estas poblaciones.

De acuerdo con Donoso (1993), N. glauca en su distribución más septentrional solo crece en la Cordillera de la Costa, sobre suelos rocosos que se caracterizan por tener pendientes fuertes. Más al Sur, el Bosque Maulino de N. glauca se desarrolla sobre granodioritas medianamente profundas y con $\mathrm{pH}$ ácido, aunque en gran parte de su superficie lo hace sobre materiales metamórficos (esquistos) (Donoso 1993). En esta cordillera crece generalmente en cerros con topografía suave a mediana, en lomajes entre 200 y $600 \mathrm{~m}$ de altitud. En la Cordillera de Los Andes, N. glauca se encuentra por lo general sobre suelos de origen volcánico o granítico, que varían según altitud y exposición. Normalmente ocupa exposiciones de solana sobre los $600 \mathrm{~m}$ hasta los $1.100 \mathrm{~m}$ de altitud. En altitudes más bajas, crece en suelos más profundos de tipo trumao y a veces se ubica en exposiciones de umbría asociándose a especies más mesófitas. Es frecuente observar bosques de esta especie en pendientes relativamente fuertes y rocosas, pero en las bajas altitudes se encuentra en lomajes suaves y áreas planas, cuando no ha sido sustituido (Donoso 1993). No hay grandes diferencias en el nivel de acidez del suelo entre la distribución de la Cordillera de la Costa y la de la Cordillera de Los Andes, encontrándose valores de $\mathrm{pH}$ del suelo entre 5,0 y 5,8 (Córdova y Pérez 1996).

\section{POBLACIONES DE NOTHOFAGUS GLAUCA ASOCIADAS A LA TIPOLOGÍA FORESTAL}

En su distribución natural, se aprecian cinco grandes áreas con bosques de $N$. glauca (figura 1). En su distribución más septentrional, en la Región Metropolitana y en la Región de O'Higgins, se encuentran algunos bosquetes de N. glauca asociado con Nothofagus macrocarpa (A.DC.)
Vazq. et Rodr. Más al sur, en su área de mayor presencia, se localiza en la formación conocida como Bosque Maulino, tanto en la Cordillera de la Costa como en la Cordillera de Los Andes, y aunque tiende a formar bosques puros se puede encontrar asociado con Nothofagus obliqua (Mirb.) Oerst., en ambas cordilleras, y con Nothofagus alessandrii Espinosa en la Cordillera de la Costa. En el rodal más meridional descrito, destacan entre las especies acompañantes $N$. obliqua y, en un dosel inferior, algunas del tipo esclerófilas (Le-Quesne y Sandoval 2001).

En Chile, la vegetación se ha clasificado en regiones y subregiones sobre la base de criterios biológicos, edáficos, y climáticos (Gajardo 1994). En esta clasificación, N. glauca se localiza en parte de la Región Bosque Caducifolio, en la Subregión de los Bosques Caducifolios Montanos, y en las formaciones vegetacionales Bosque Caducifolio Maulino y Bosque Caducifolio de la Pre-Cordillera. Sobre esta base, e integrando otras componentes, Luebert y Pliscoff (2004) propusieron una clasificación en pisos de vegetación, ubicando a $N$. glauca en el Matorral y Bosque Caducifolio, en el cual se pueden distinguir tres asociaciones:

- Nothofagus glauca - Azara petiolaris, que es una formación boscosa dominada por las especies arbóreas principales N. glauca, Gevuina avellana Mol., Lomatia hirsuta (Lam.) Diels ex Macbr. y Azara petiolaris (D. Don) Johnst.

- Bosque caducifolio mediterráneo costero de Nothofagus glauca y Persea lingue, que se caracteriza por ser un bosque caducifolio dominado por N. glauca, N. obliqua, G. avellana y Persea lingue (R. et P.) Nees ex Kopp, con Gaultheria insana (Mol.) D.J. Middleton, Ugni molinae Turcz. y Escallonia pulverulenta (R. et P.) Pers. como diferenciales de la estrata arbustiva.

- Bosque caducifolio mediterráneo andino de Nothofagus glauca y Nothofagus obliqua, que corresponde a un bosque caducifolio en el que dominan $N$. glauca y N. obliqua, formando por lo general bosques espacialmente segregados y solo se mezclan en las exposiciones más húmedas.

Si bien es cierto que estos pisos vegetacionales comprenden el área de distribución natural de $N$. glauca, hay zonas que no están cubiertas y que corresponden a pisos del tipo bosque esclerófilo mediterráneo. Esto se da en parte de la distribución costera en el norte de la región del Maule y en todos aquellos bosques más septentrionales, presentes en las regiones de O'Higgins y Metropolitana. Esto podría ser, además, un elemento diferenciador de zonas de crecimiento.

\section{DIFERENCIACIÓN GENECOLÓGICA. VARIACIÓN CLINAL O ECOTÍPICA}

La distribución geográfica latitudinal de las especies del género Nothofagus en Chile y la variación de la preci- 
pitación, tanto latitudinal como longitudinal, son factores que facilitan la diferenciación genecológica de las especies (Donoso 1987). En su distribución natural, N. glauca crece en variadas condiciones de sitio. Si bien es cierto que no existe una marcada diferencia en los tipos de suelo y que, por lo general, se encuentra en exposiciones de solana en laderas con pendientes suaves o fuertes, a lo largo de la gradiente latitudinal en los más de $330 \mathrm{~km}$ que separan la población más septentrional de la más meridional, se presentan diferentes condiciones climáticas, con marcadas diferencias, por ejemplo, en el nivel de precipitaciones.

Que en toda el área de su distribución natural las condiciones ambientales varíen considerablemente podría favorecer la diferenciación genecológica de las poblaciones de $N$. glauca. En este contexto, se podría esperar una diferenciación ecotípica o del tipo clinal, como sucede con otras especies de Nothofagus (Donoso 1987) que, en parte, crecen en una zona similar y contigua a la de $N$. glauca (e.g. Nothofagus dombeyi (Mirb.) Oerst). Sin embargo, no se han encontrado reportes que confirmen esta hipótesis. No obstante, el tamaño de las semillas podría estar condicionado por la procedencia; la semilla de árboles de la distribución andina en la región del Maule, tienden a ser más largas y de menor peso que las de la distribución costera en la misma región, aunque su capacidad germinativa, en torno a un $40 \%$ de las semillas viables, es similar en ambos sectores (Santelices et al. 2017). Según Santelices et al. (2017), si bien no se ha reportado una relación positiva entre el peso de la semilla y su capacidad germinativa, es destacable el hecho de que en semillas de procedencias andinas, con un peso promedio de 1.000 semillas igual a $427,7 \pm 9,3 \mathrm{~g}$, se haya registrado la capacidad germinativa más baja (menor al $30 \%$ ), situación ocurrida en las poblaciones de Vilches y El Melado, localizadas en la Cordillera de Los Andes en la región del Maule, en altitudes cercanas a 1.000 m s.n.m.; por el contrario, en procedencias de la costa, se registró un peso promedio de 1.000 semillas igual a $482,8 \pm 13$ g y una capacidad germinativa homogénea para todas las poblaciones analizadas $(40,21 \pm 3,5 \%)$. Es posible que gran parte de esta variabilidad de tipo discontinua o ecotípica, sea producto de la clara interrupción de la continuidad de la especie entre ambas cordilleras, además de diferencias climáticas y altitudinales. De lo contrario, la especie presentaría una amplia plasticidad fenotípica para el carácter peso de la semilla. No obstante, dado el rango de distribución de la especie, estos son aspectos que debieran ser investigados en mayor profundidad.

En cuanto a la ciclicidad en la producción de semillas, $N$. glauca tiene un comportamiento similar al de otras especies del género (Monks y Kelly 2006), siendo también clasificada como una especie vecera. No obstante, no se han caracterizado los ciclos de semillación. Considerando la importancia de la especie y la información insuficiente para optimizar la toma de decisiones orientadas al manejo en términos de conservación o producción, deberían profundizarse este tipo de estudios y analizarse el compor- tamiento en la producción de las semillas en una escala temporal mayor.

\section{VARIACIÓN EN CRECIMIENTO}

Si bien el territorio en donde se distribuye $N$. glauca no es tan extenso como el de otras especies del género (Donoso 1987), las variadas condiciones de sitio deberían reflejarse en diferentes zonas de crecimiento. Sin embargo, esto no se ha verificado dado que los estudios sobre el crecimiento de la especie son reducidos.

Aunque no existen reportes sobre el crecimiento inicial de esta especie, existen algunas investigaciones en rodales maduros realizadas mediante la técnica del análisis de tallo o fustal, en diferentes sitios de la distribución andina de $N$. glauca, observándose áreas basales que varían entre 40 $\mathrm{m}^{2} \mathrm{ha}^{-1}$ y $61,5 \mathrm{~m}^{2} \mathrm{ha}^{-1}$ (Mollenhauer 1975, Donoso 1988). Un rodal que tenía 71 años de edad y $504 \mathrm{~m}^{3}$ ha-1 de volumen presenta la mayor área basal $\left(61,5 \mathrm{~m}^{2} \mathrm{ha}^{-1}\right)$, localizado en el sector de Radal Siete Tazas, en la Cordillera de Los Andes en la provincia de Talca de la región del Maule, cuyo crecimiento medio anual observado en los árboles dominantes fue de $0,43 \mathrm{~cm}$ en diámetro medio cuadrático y $0,36 \mathrm{~m}$ en altura (Donoso 1988). En cambio, en un bosque más joven (38 años de edad regenerado por monte bajo), con $40 \mathrm{~m}^{2}$ ha-1 de área basal, localizado un poco más al Sur, pero siempre en la distribución andina (Bullileo, en la provincia de Linares en la región del Maule), el volumen observado fue de $180 \mathrm{~m}^{3} \mathrm{ha}^{-1}$ (Mollenhauer 1975). En estos dos bosques, el ritmo de crecimiento disminuyó fuertemente con la edad, llegando a valores de un $50 \%$ en volumen y área basal al compararlos con el de los primeros años.

Con la información generada mediante análisis fustales en rodales localizados en Radal Siete Tazas y Bullileo (Mollenhauer 1975, Donoso 1988), se desarrolló un modelo que puede estimar la altura y la edad de los árboles dominantes en función del diámetro para esa zona de crecimiento, pudiéndose apreciar algunos valores en el cuadro 2 (Santelices y Stephan 1998).

En cuanto al cultivo de plantas en vivero, existe información suficiente para producir plantas en una temporada con una calidad funcional aceptable para su posterior establecimiento en campo (Santelices et al. 2013ab, Cabello et al. 2019). No obstante, solo se ha reportado una experiencia sobre plantaciones de N. glauca (Quiroz et al. 2009), que en definitiva es la instancia para evaluar la calidad de las mismas. En el proceso de producción de plantas en la Zona Central de Chile, se ha observado que es necesario protegerlas de la radiación directa (Santelices et al. 2013a), siendo usualmente utilizadas para este efecto mallas de sombra, ampliamente aceptadas como medio de cultivo bajo condiciones ambientales semicontroladas. Aún no se han establecido atributos morfológicos para la comercialización del material de propagación de uso forestal para $N$. glauca, pero se podrían utilizar los em- 
pleados para Nothofagus alpina (Poepp. et Endl.) Oerst. (INN 2005). Esta norma considera una planta de calidad suficiente aquella que tiene entre 25 y $35 \mathrm{~cm}$ de altura y un diámetro de cuello mayor a $3 \mathrm{~mm}$ y que ha sido cultivada a raíz cubierta durante una temporada; en el caso de aquellas cultivadas a raíz desnuda también durante una temporada, los requerimientos son dimensiones entre 25 y $40 \mathrm{~cm}$ de altura y un diámetro de cuello mayor a $5 \mathrm{~mm}$ (INN 2005). En general, la información recogida en los reportes sobre producción de plantas de $N$. glauca se encuentra en los rangos sugeridos para esas condiciones, especialmente para aquellas cultivadas a raíz cubierta (cuadro 3).

Si bien es cierto que las plantas cultivadas durante una temporada (1/0) a raíz desnuda en general no alcanzan las dimensiones en altura y diámetro sugeridas por la norma chilena antes mencionada, sí están en el rango propuesto para la relación entre esos dos atributos, con un índice de esbeltez levemente inferior a 5. En cambio, aquellas cultivadas a raíz cubierta, también del tipo $1 / 0$, tanto en su diámetro como en la esbeltez de su tallo, están en los rangos sugeridos por la norma para plantas de buena calidad. En ambientes mediterráneos, caracterizados por un periodo prolongado sin precipitaciones, como donde se distribuye naturalmente $N$. glauca, la tendencia es producir plantas a raíz cubierta. Por otra parte, el índice de esbeltez observado en las plantas se encuentra en el rango considerado como aceptable para especies de climas mediterráneos (Villar-Salvador 2003). En cuanto a la relación de la biomasa aérea con la biomasa radical (índice tallo/raíz), si bien es cierto que no existe mucha información sobre el particular para climas mediterráneos, se han sugerido valores entre 1,5 y 2,2 (Villar-Salvador 2003); en las experiencias reportadas para $N$. glauca se observan valores en los índices de calidad que podrían indicar que las plantas tendrían en campo un adecuado balance hídrico (cuadro 3).

En cuanto al crecimiento de $N$. glauca en plantaciones, solo existe un reporte (Quiroz et al. 2009), en el cual se analizó el efecto del volumen radical, de la protección lateral con malla plástica, y del riego, sobre el desarrollo inicial de esta especie. Estos autores concluyen que, culminada la segunda temporada de crecimiento, no hay un efecto sobre el crecimiento diametral como consecuencia del volumen de raíces de las plantas, aunque el diámetro de aquellos individuos sin protección fue significativamente mayor; no obstante, en aquellas plantas bajo protección con malla se registraron las mayores alturas; en cuanto a la

Cuadro 2. Altura y edad en función del DAP de árboles dominantes de Nothofagus glauca de dos sitios con diferente área basal en la distribución andina de la especie (Bullileo con $40 \mathrm{~m}^{2} \mathrm{ha}^{-1}$ y Radal Siete Tazas con 61,5 $\mathrm{m}^{2} \mathrm{ha}^{-1}$ ) (Santelices y Stephan 1998).

Height and age as a function of DBH of dominant Nothofagus glauca trees from two sites with different basal area in the Andean distribution of the species (Bullileo with $40 \mathrm{~m}^{2} \mathrm{ha}^{-1}$ and Radal Siete Tazas with $61.5 \mathrm{~m}^{2} \mathrm{ha}^{-1}$ ) (Santelices and Stephan 1998).

\begin{tabular}{ccccccccc}
\hline \multirow{2}{*}{$\begin{array}{c}\text { Área Basal } \\
\left(\mathrm{m}^{2} \mathrm{ha}^{-1}\right)\end{array}$} & & \multicolumn{7}{c}{ Edad (años) } \\
\cline { 3 - 9 } 40,0 & Altura (m) & 3,1 & 7,0 & 10,6 & 13,7 & 16,6 & 19,1 & 21,2 \\
& DAP (cm) & 4,9 & 9,4 & 13,1 & 16,3 & 19,1 & 21,6 & 23,9 \\
\hline \multirow{2}{*}{61,5} & Altura (m) & 5,2 & 10,5 & 14,9 & 18,5 & 21,4 & 23,7 & 25,3 \\
& DAP (cm) & 6,0 & 11,3 & 16,1 & 20,3 & 24,1 & 27,4 & 30,2 \\
\hline
\end{tabular}

Cuadro 3. Efecto de diferentes tratamientos culturales en vivero sobre los atributos de plantas de Nothofagus glauca cultivadas durante una temporada (Santelices et al. 2013ab, Cabello et al. 2019).

Effect of different cultural treatments in nursery on the attributes of Nothofagus glauca plants grown during a season (Santelices et al. 2013ab, Cabello et al. 2019).

\begin{tabular}{|c|c|c|c|c|c|}
\hline \multirow{2}{*}{ Tratamiento } & \multirow{2}{*}{$\begin{array}{l}\text { Longitud del } \\
\text { tallo }(\mathrm{cm})\end{array}$} & \multirow{2}{*}{$\begin{array}{l}\text { Diámetro de cuello } \\
\text { de la raíz }(\mathrm{mm})\end{array}$} & \multirow{2}{*}{$\begin{array}{l}\text { Biomasa } \\
(\mathrm{g})\end{array}$} & \multicolumn{2}{|c|}{ Índices de calidad } \\
\hline & & & & Esbeltez & Tallo/Raíz \\
\hline Estratificación - 60 días (raíz desnuda) & 22 & 4,7 & 0,4 & 4,6 & 1,8 \\
\hline $\mathrm{GA}_{3}-800 \mathrm{mg} \mathrm{L}{ }^{-1}$ (raíz desnuda) & 16 & 4,1 & 1,2 & 3,9 & 1,0 \\
\hline Sombra $-50 \%$ (raíz desnuda) & 15 & 3,2 & - & 4,7 & - \\
\hline Siembra en septiembre (raíz desnuda) & 10 & 3,0 & 0,4 & 3,4 & 0,9 \\
\hline $\mathrm{GA}_{4}-100 \mathrm{mg} \mathrm{L}^{-1}$ (raíz cubierta) & 29 & 4,5 & - & 6,4 & - \\
\hline Sombra $18-35 \%$ (raíz cubierta) & 18,5 & 3,0 & 2,6 & 6,3 & 0,9 \\
\hline Fertilización Basacote $^{\circledR} 7,5-10,0 \mathrm{~g} \mathrm{~L}^{-1}$ (raíz cubierta) & 22,9 & 3,1 & 3,1 & 8,0 & 1,1 \\
\hline
\end{tabular}


supervivencia, siempre fue mayor en aquellas con protección lateral. Esto último confirmaría, en parte, que $N$. glau$c a$ sigue una estrategia de regeneración por claros (Donoso 1993), necesitando de un espacio al interior del bosque con cierto grado de luminosidad, pero con sombra que ejerza una cierta protección lateral.

\section{VARIACIÓN DEBIDO A HIBRIDACIÓN E INTROGRESIÓN}

Un factor importante de variación en las poblaciones de $N$. glauca se produce por la hibridación de esta especie con N. obliqua, la que genera el híbrido Nothofagus leonii Espinosa (huala), el cual se retrocruza con sus padres (Donoso y Landrum 1979). Las evidencias indican la tendencia a una infiltración de genes de $N$. glauca en las poblaciones de $N$. obliqua más que al revés, pero en los individuos introgresantes pueden aparecer muchas características de $N$. glauca que, a veces, hacen difícil distinguir con claridad a un $N$. glauca de un híbrido, elemento que claramente es un factor de variabilidad tanto para $N$. glauca como para $N$. obliqua (Santelices et al. 2013a). La variabilidad debida a la introgresión también se aprecia en la madera (Donoso y Landrum 1979).

La hibridación entre N. obliqua y N. alpina, que produce el híbrido natural reconocido hoy como rorra, se produce en parte del área que lo hace $N$. leonii. Es posible, entonces, el cruzamiento entre un híbrido rorra con N. glauca, lo que podría significar otro factor de variación para esta especie (Donoso et al. 2004).

\section{VARIACIÓN GENÉTICA DETECTADA MEDIANTE MARCADORES ISOENZIMÁTICOS Y MOLECULARES}

La variabilidad genética o diversidad genética, en sentido amplio, es el componente más básico de la biodiversidad, y los efectos de los tres principales procesos destructivos (i.e., deforestación, tala indiscriminada y fragmentación) sobre la diversidad genética pueden abarcar desde impactos negativos en la reproducción y supervivencia de una especie, hasta barreras para el intercambio migratorio y su extinción total o parcial. Según Bustamante et al. (2005), los efectos de la fragmentación del Bosque Maulino chileno, ecosistema al cual pertenece $N$. glauca, podrían llevar a una disminución de la abundancia de la especie, de ahí que el conocimiento de su diversidad genética sea una herramienta vital para la conservación y manejo de su patrimonio genético.

En el caso de $N$. glauca, y al igual que para $N$. alessandrii, la información sobre su diversidad genética es escasa. No obstante, y debido a su distribución geográfica, es esperable que la especie contenga altos niveles de diversidad genética y sea menos afectada por efectos estocásticos que producen su deterioro (e.g., deriva génica). Además, dado su sistema de polinización por viento y fecundación cruzada (Ipinza y Espejo 2000), se podría esperar que la diferenciación genética sea mayor al interior de las poblaciones, al igual que en N. alpina (Marchelli y Gallo 2001). Sin embargo, el alto nivel de fragmentación de la especie, debido a la baja producción y alta depredación de sus semillas (Donoso et al. 2003b, Burgos et al. 2008), el bajo reclutamiento de plantas (Castillo 2005) y el aislamiento entre algunas poblaciones (Vergara et al. 2014), son factores que a futuro podrían afectar negativamente los niveles de diversidad genética de la especie.

Martín et al. (2010) desarrollaron marcadores a base de las proteínas de reserva de la semilla. Los autores muestrearon una población costera (Reserva Los Queules, $35^{\circ}$ $58^{\prime} \mathrm{S}-72^{\circ} 41^{\prime} \mathrm{O}$ ) y una precordillerana (San Fabián de Alico, $36^{\circ} 33^{\prime} \mathrm{S}-71^{\circ} 32^{\prime} \mathrm{O}$ ), analizando cinco semillas por árbol (no se informa la cantidad de árboles muestreados por población). Se encontró un nivel de diversidad genética medio a alto $\left(\mathrm{H}_{\mathrm{E}}=0,241\right)$ y una similitud genética entre ambas poblaciones. Más tarde, en un estudio utilizando marcadores de tipo microsatélite (Vergara et al. 2014), se analizaron siete loci en una muestra de ocho poblaciones costeras y precodilleranas de $N$. glauca, utilizando 16 individuos por población y abarcando desde los $34^{\circ} 10^{\prime}$ ' a los $37^{\circ} 28^{\prime}$ de latitud Sur y $71^{\circ} 01^{\prime}$ a $72^{\circ} 40^{\prime}$ de longitud Oeste. Los resultados indican que, a pesar de la explotación, sustitución y fragmentación de los bosques de la especie, esta mantiene una alta diversidad genética. Se encontró un promedio de 4,5 alelos por locus (A) y el parámetro $\mathrm{H}_{\mathrm{E}}$ alcanzó una media de 0,50. Estos valores, son inferiores a los reportados en el mismo estudio para las especies de más amplio rango $N$. obliqua $\left(\mathrm{A}=6,2, \mathrm{H}_{\mathrm{E}}=0,66\right)$ y $N$. alpina $\left(\mathrm{A}=5,5, \mathrm{H}_{\mathrm{E}}=0,62\right)$, lo que muestra una correlación positiva entre los niveles de diversidad genética y la extensión de la distribución geográfica en especies relacionadas. Un resultado que requiere ser profundizado es la presencia de coeficientes de endogamia que, aunque no estadísticamente significativo, son o bien cercanos a cero o positivos $\left(\mathrm{F}_{\text {IS }}=\right.$ $-0,007$ a 0,215$)$. Es interesante el hecho de que el valor más alto para el parámetro $\mathrm{F}_{\mathrm{IS}}$ se encuentre en la población denominada Siete Tazas $\left(\mathrm{F}_{\mathrm{IS}}=0,215\right)$, perteneciente a la Reserva Nacional Radal Siete Tazas en la región del Maule, es decir, bajo un sistema estatal de protección. Si bien la endogamia debería ser más común en las poblaciones de $N$. glauca que están más aisladas geográficamente y que son más pequeñas, estos resultados podrían indicar que en las áreas protegidas solo se están conservando poblaciones históricamente marginales y que el proceso de fragmentación en las poblaciones del centro de la distribución es muy reciente como para que se refleje en una endogamia acentuada. Este es un aspecto que debería ser investigado en mayor profundidad.

Desde el punto de vista de la estructura poblacional, el nivel de diferenciación al interior de las poblaciones de $N$. glauca es alto (91\%), en tanto que la diferenciación genética entre poblaciones es baja $(8,7 \%)$ (Vergara et al. 2014), lo que es típico en especies forestales e indica que 
la mayoría de la diversidad genética está al interior de las poblaciones y que hay un moderado a alto flujo de genes entre poblaciones, mediado por la dispersión del polen. Así, la tasa de migración efectiva (i.e., parámetro $\mathrm{N}_{\mathrm{e}} \mathrm{m}$ ) tiene un valor de 2,6 individuos por generación, lo que es suficiente para mantener a las poblaciones fuera del riesgo de deriva genética (Vergara et al. 2014). Además, los autores indican que las poblaciones pueden dividirse en tres grupos. El primer grupo incluye poblaciones costeras y precordilleranas ubicadas aproximadamente entre los $35^{\circ}$ a $36,3^{\circ}$ de latitud Sur y $71^{\circ}$ a $72,4^{\circ}$ de longitud Oeste, similar a lo reportado por Martín et al. (2010), en tanto que en el segundo y el tercer grupo quedarían las poblaciones aisladas de Loncha por el Norte $\left(34^{\circ} 10^{\prime}-71^{\circ} 01^{\prime}\right)$ y Quilleco por el Sur $\left(37^{\circ} 28^{\prime}-71^{\circ} 58^{\prime}\right)$, respectivamente (figura $2 \mathrm{~A})$. Complementariamente, en la figura $2 \mathrm{~B}$ se muestra que el segundo y tercer grupo tienen una identidad genética relativamente diferenciada el uno del otro, y que las poblaciones del primer grupo son una combinación balanceada de ambas identidades. Dentro del primer grupo, la similitud genética entre las poblaciones de Tregualemu (costa) y San Fabián de Alico (precordillera) indicaría que el flujo de genes tiene una aparente orientación Este-Oeste, lo que podría explicarse por la orientación de las cadenas montañosas en la zona.

Los antecedentes mencionados indican que el estado de conservación genética de $N$. glauca no es aún crítico y que su nivel de diversidad neutral es medio a alto. No obstante, la baja representatividad de la especie en el Sistema Nacional de Áreas Silvestres Protegidas (SNASPE), solo un 2 \% (Ormazábal y Benoit 1987) y la fuerte presión antropogénica, son factores que podrían conducir a la especie a un mayor grado de deterioro en el mediano o largo plazo. A lo anterior se suma la nula información acerca del potencial adaptativo de la especie y su comparación con la diversidad neutral (i.e., comparaciones $\mathrm{Q}_{\mathrm{ST}} \mathrm{vs}_{\mathrm{ST}}$ ). Santelices et al. (2017), al analizar la capacidad germinativa y supervivencia de poblaciones costeras y precordilleranas de $N$. glauca, encontraron una mayor diferenciación al interior de las poblaciones que entre poblaciones; las poblaciones precordilleranas tendrían una menor capacidad germinativa y supervivencia. Similares resultados fueron reportados por Santelices et al. (2013a) y podrían estar relacionados con la distribución altitudinal de estas
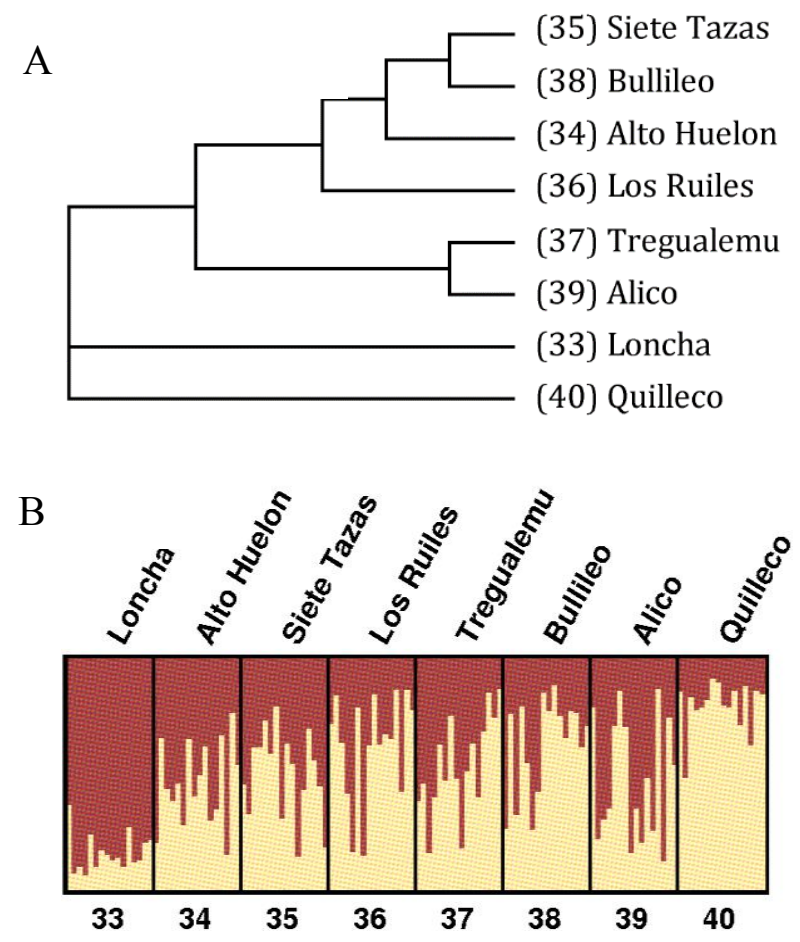

Figura 2. A) Dendrograma obtenido usando valores de diferenciación genética entre todos los pares de poblaciones de Nothofagus glauca estudiadas por Vergara et al. (2014). B) Representación gráfica de la estructura encontrada entre las poblaciones de Nothofagus glauca estudiadas por Vergara et al. (2014). El análisis muestra dos probables identidades genéticas y un alto flujo de genes entre poblaciones (los grupos de poblaciones están representados por colores, las poblaciones están definidas por líneas verticales y ordenadas de norte a sur, y dentro de los individuos, la proporción de cada color indica pertenencia al grupo dado).

A) Dendrogram obtained using genetic differentiation values between all pairs of Nothofagus glauca populations studied by Vergara et al. (2014). B) Graphic representation of the structure found among the populations of Nothofagus glauca studied by Vergara et al. (2014). The analysis shows two probable genetic identities and high gene flow among populations (groups of populations are represented by colors, populations are defined by vertical lines and ordered from north to south, and within individuals, the proportion of each color indicates membership in the given group). 
poblaciones. Además, como se mencionó anteriormente, $N$. glauca tiene un bajo nivel de reclutamiento de plantas, situación que disminuiría a medida que se avanza hacia el límite altitudinal de su distribución, dados los estreses ambientales a los que se ve sometida la especie.

Las zonas con una alta diversidad genética son las más importantes para la conservación de una especie, ya que constituyen el material base tanto para programas de mejoramiento como para el cultivo de la especie fuera de su área de distribución. Los esfuerzos de conservación debieran concentrase en las poblaciones genéticamente distintivas, y en poblaciones con mayor riqueza alélica global de las especies. De estas poblaciones, la población aislada de Quilleco en el límite sur de la distribución de esta especie, tiene la primera prioridad debido a su vulnerabilidad, luego San Fabián de Alico, y finalmente Roblería Cobre de Loncha y Los Ruiles, poblaciones actualmente protegidas pero que requieren la protección de poblaciones aledañas complementarias para disminuir la probabilidad de extinción debido especialmente a incendios forestales. Además, si bien las poblaciones ubicadas en las Reservas Nacionales Los Ruiles, Altos de Lircay, Radal Siete Tazas y Roblería Cobre de Loncha están protegidas, los resultados reportados por Vergara et al. (2014) coinciden en que en las áreas protegidas habría niveles de endogamia más altos, por lo que se debería profundizar la estrategia adecuada para enriquecer su acervo genético. Para esto, otra tarea a futuro es la evaluación de la diversidad genética de la especie a partir de ADN cloroplástico, con el propósito de conocer la composición de haplotipos poblacionales y planificarla transferencia de material genético de manera adecuada. A modo de ejemplo, Marchelli et al. (1998) reportaron distintos haplotipos para N. alpina en Argentina, los cuales mostraban una notoria separación Norte-Sur. Esto permitiría evitar alterar negativamente la identidad y diversidad genética de la especie al momento de llevar a cabo labores de restauración.

\section{CONSIDERACIONES FINALES}

La información presentada evidencia la variabilidad intra-específica en múltiples rasgos de $N$. glauca. Los hechos descritos previamente en relación con su distribución (espacial, ecológica y en relación con tipología forestal) demuestran que $N$. glauca está sometido a diferentes condiciones climáticas, lo que sugiere variabilidad fenotípica y genotípica. Esta variabilidad es corroborada, además, a través de estudios genéticos. Las investigaciones a la fecha, valiosas como línea base, permiten planificar una segunda fase, de tipo experimental, la cual debiera comparar los distintos genotipos y/o fenotipos en condiciones ambientales extremas y posibles en escenarios de cambio climático. De esta forma se podrán seleccionar poblaciones que puedan hacer frente a futuras, pero próximas, condiciones de sequía y/o de aumento de temperatura, enfermedades, entre otras. Otro punto a considerar para futuros estudios, es el microbioma asociado a esta especie vegetal (Trivedi et al. 2020). Por ejemplo, las raíces de las plantas del género Nothofagus forman asociaciones simbióticas obligadas con una gran cantidad de especies de hongos del suelo, generando un órgano clave para la nutrición y tolerancia a estresores bióticos y abióticos. Este órgano conocido como ectomicorriza constituye una fuente de variación importante que también permitirá seleccionar individuos capaces de lidiar de mejor forma con un futuro escenario de cambio climático. Por lo tanto, es urgente el estudio próximo en situaciones experimentales y que consideren el microbioma de $N$. glauca de forma tal de poder entregar herramientas concretas para los programas de conservación y restauración de bosques de esta especie.

\section{REFERENCIAS}

Arroyo MTK, M Riveros, A Peñaloza, L Cavieres, AM Faggi. 1996. Phytogeographic relationships and regional richness patterns of the cool temperate rainforest flora of southern South America. In Lawford RG, PB Alaback, E Fuentes eds. High-Latitude rainforests and associated ecosystems of the west Coasts of the Americas: climate, hydrology, ecology and conservation. New York. USA. Springer. p. 134-172.

Barstow M, MC Rivers, H Baldwin. 2017. Nothofagus glauca. The IUCN Red List of Threatened Species 2017: e.T32034A2809142. Consultado ene. 2018. Disponible en https://dx.doi.org/10.2305/IUCN.UK.2017-3.RLTS. T32034A2809142.en.

Burgos A, AA Grez, RO Bustamante. 2008. Seed production, pre-dispersal seed predation and germination of Nothofagus glauca (Nothofagaceae) in a temperate fragmented forest in Chile. Forest Ecology and Management 255: 12261233. DOI: https://doi.org/10.1016/j.foreco.2007.10.032.

Bustamante R, A Grez, J Simonetti, J San Martín. 2005. La fragmentación del bosque Maulino y su dinámica regeneracional: diagnóstico actual y perspectivas futuras. In Smith C, J Armesto, C Valdovinos eds. Historia, Biodiversidad y Ecología de los Bosques de la Cordillera de la Costa. Santiago, Chile. Editorial Universitaria. p. 529-539.

Bustamante R, J Simonetti. 2005. Is Pinus radiata invading the native vegetation in Central Chile? Demographic responses in a fragmented forest. Biological Invasions 7: 243-249. DOI: https://doi.org/10.1007/s10530-004-0740-5.

Cabello A, N Espinoza, S Espinoza, A Cabrera, R Santelices. 2019. Effect of pre-germinative treatments on Nothofagus glauca seed germination and seedling growth. New Zealand Journal of Forestry Science 49: 1-9. DOI: https://doi. org/10.33494/nzjfs492019x34x.

Castillo J. 2005. Tasa de reclutamiento y mortalidad de especies arbóreas en el Bosque Maulino costero: efecto del intervalo censal, la fragmentación del bosque y la especie. Tesis Ingeniería en Recursos Naturales Renovables. Santiago, Chile. Facultad de Ciencias Agronómicas, Universidad de Chile. 34 p.

Córdova D, M Pérez. 1996. Estudio comparativo de las comunidades de "hualo" (Nothofagus glauca (Phil). Krasser) en el sector costero y la precordillera de la VII Región. Taller de 
Habilitación Profesional. Talca, Chile. Universidad Católica del Maule. 49 p.

Donoso C. 1987. Variacion natural en especies de Nothofagus en Chile. Bosque 8(2): 85-97. DOI: https://doi.org/10.4206/ bosque.1987.v8n2-03

Donoso C. 1993. Bosques templados de Chile y Argentina. Variación, estructura y dinámica. Santiago, Chile. Editorial Universitaria. $484 \mathrm{p}$.

Donoso C, L Gallo, PJ Donoso, M Azpilicueta. 2004. Variación en Nothofagus obliqua (Mirb.) Oerst. (Roble, Coyán, Hualle o Pellín). In Donoso C, AC Premoli, L Gallo, R Ipinza eds. Variación intraespecífica en especies arbóreas de los bosques templados de Chile y Argentina. Santiago, Chile. Editorial Universitaria. p. 79-113.

Donoso C, L Landrum. 1979. Hybridization and gynecological differentiation in chilean Nothofagus. Archivos de Biologia y Medicina Experimentales 12: 634-634.

Donoso P. 1988. Caracterización, crecimiento y proposiciones silviculturales para comunidades de Nothofagus en el Area de Protección "Radal - 7 Tazas", VII Región. Tesis Ingeniero Forestal. Valdivia, Chile. Facultad de Ciencias Forestales, Universidad Austral de Chile. 101 p.

Gajardo R. 1994. La Vegetación Natural de Chile. Clasificación y Distribución Geográfica. Santiago, Chile. Editorial Universitaria. $165 \mathrm{p}$.

Hijmans RJ, SE Cameron, JL Parra, PG Jones, A Jarvis. 2005. Very high resolution interpolated climate surfaces for global land areas. International Journal of Climatology 25: 1965-1978. DOI: https://doi.org/10.1002/joc.1276.

INN (Instituto Nacional de Normalización, CL). 2005. NCH2957/5 Madera. Material de propagación de uso forestal. Parte 5: requisitos generales para raulí. Santiago, Chile. Instituto Nacional de Normalización (INN). 6 p.

Ipinza R, J Espejo. 2000. Biología reproductiva en Nothofagus. In Ipinza R, B Gutiérrez, V Emhart eds. Domesticación y mejora genética de raulí y roble. Valdivia, Chile. Universidad Austral de Chile- Instituto Forestal. p. 75-93.

Le-Quesne C, L Sandoval. 2001. Extensión del límite sur para Nothofagus glauca (Phil.) Krasser. Gayana Botánica 58: 139-142. DOI: http://dx.doi.org/10.4067/S0717-66432001000200005.

Litton CM, R Santelices. 2003. Effect of wildfire on soil physical and chemical properties in a Nothofagus glauca forest, Chile. Revista Chilena de Historia Natural 76: 529-542. DOI: http://dx.doi.org/10.4067/S0716-078X2003000400001.

López E. 1994. Caracterización bioclimática de la estación meteorológica de la Universidad Católica del Maule. Taller de Habilitación Profesional. Talca, Chile. Facultad de Ciencias Agrarias y Forestales, Universidad Católica del Maule. 50 p.

Luebert F, P Pliscoff. 2004. Clasificación de pisos de vegetación y análisis de representatividad ecológica para áreas propuestas para protección de la ecorregión. Valdivia, Chile. WWF Chile. 174 p.

Marchelli P, LA Gallo. 2001. Genetic diversity and differentiation in a southern beech subjected to introgressive hybridization. Heredity 87: 284-293. DOI: https://doi.org/10.1046/ j.1365-2540.2001.00882.x.

Marchelli P, LA Gallo, F Scholz, B Ziegenhagen. 1998. Chloroplast DNA markers reveal a geographical divide across Argentinean southern beech Nothofagus nervosa (Phil.) Dim. et Mil. distribution area. Theoretical and Applied Genetics 97: 642-646. DOI: https://doi.org/10.1007/s001220050940.
Martín MA, S Muñoz, F Muñoz, M Uribe, JR Molina, MA Herrera, LM Martín, JB Alvarez. 2010. Primeros resultados en el desarrollo de un marcador genético basado en las proteínas de reserva en dos especies del género Nothofagus. Bosque 31(3): 252-257. DOI: http://dx.doi.org/10.4067/ S0717-92002010000300010.

MSEGPRES (Ministerio Secretaría General de la Presidencia, CL). 2008. Decreto Supremo 51. 2008. Diario Oficial de la República de Chile, $\mathrm{N}^{\circ}$ 39.100. http://www. mma.gob.cl/clasificacionespecies/Anexo tercer_proceso/ DS 51_2008_3erProcesoClasif_completo.pdf

Mollenhauer K. 1975. Estudio de estructura en renovales de Nothofagus glauca (Phil.) Krasser, en Bullileo. Tesis Ingeniero Forestal. Santiago, Chile. Facultad de Ciencias Forestales, Universidad de Chile. 63 p.

Monks A, D Kelly. 2006. Testing the resource-matching hypothesis in the mast seeding tree Nothofagus truncata ( $\mathrm{Fa}$ gaceae). Austral Ecology 31: 366-375. DOI: https://doi. org/10.1111/i.1442-9993.2006.01565.x.

Ormazábal C, I Benoit. 1987. El estado de conservación del género Nothofagus en Chile. Bosque 8(2): 109-120. DOI: https://doi.org/10.4206/bosque.1987.v8n2-06

Quiroz I, M Pincheira, A Hernández. 2009. Restauración ecológica en la zona central de Chile: técnicas silvícolas para la supervivencia y crecimiento de ruil, hualo, y peumo bajo condiciones de estrés hídrico. Chile Forestal 363: 50-53.

Rioseco R, C Tesser. 2016. Cartografía interactiva de los climas de Chile. Consultado ene. 2016. Disponible en http://www7. uc.cl/sw educ/geografia/cartografiainteractiva/index.htm.

Rodríguez R, M Quezada. 2003. Fagaceae. In Marticorena C, R Rodríguez eds. Flora de Chile 2. Concepción, Chile. Universidad de Concepción. p. 64-76.

Santelices R, C Donoso, A Cabello. 2013a. Nothofagus glauca (Phil.) Krasser, Hualo, Roble maulino, Roble colorado (Maule). Familia: Nothofagaceae. In Donoso C ed. Las Especies Arbóreas de los Bosques Templados de Chile y Argentina: Autoecología. Valdivia, Chile. Marisa Cuneo Ediciones. p. 433-442.

Santelices R, S Espinoza, A Cabrera-Ariza, K Peña-Rojas, SR Donoso. 2013b. Effect of shading and fertilisation on the development of container-grown Nothofagus glauca seedlings, a threatened species from central Chile. Southern Forests 75: 145-148. DOI: 10.2989/20702620.2013.816234.

Santelices R, S Espinoza, C Magni, A Cabrera, S Donoso, K Peña. 2017. Variability in seed germination and seedling growth at the intra- and inter-provenance levels of Nothofagus glauca (Lophozonia glauca), an endemic species of Central Chile. New Zealand Journal of Forestry Science

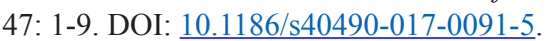

Santelices R, G Stephan. 1998. Nothofagus glauca (Phil.) Krasser. In Shutt P, HJ Schuck, UM Lang, A Roloff eds. Enzyklopädie der Holzgewächse: Handbuch und Atlas der Dendrologie. Hamburg, Deutschland. Ecomed Verlagsgesellschaft. p. 1-6.

SINIA (Sistema Nacional de Información Ambiental, CL). 2019. Sistema Nacional de Información Ambiental. Consultado ene. 2019. Disponible en http://ide.mma.gob.cl/.

Trivedi P, JE Leach, SG Tringe, T Sa, BK Singh. 2020. Plant-microbiome interactions: from community assembly to plant health. Nature Reviews Microbiology. DOI: https://doi. org/10.1038/s41579-020-0412-1 
Valencia D, J Saavedra, J Brull, R Santelices. 2018. Severidad del daño causado por los incendios forestales en los bosques remanentes de Nothofagus alessandrii Espinosa en la región del Maule de Chile. Gayana Botánica 75: 531-534. DOI: http://dx.doi.org/10.4067/S0717-66432018000100531.

Vergara R, MA Gitzendanner, DE Soltis, PS Soltis. 2014. Population genetic structure, genetic diversity, and natural history of the South American species of Nothofagus subgenus
Lophozonia (Nothofagaceae) inferred from nuclear microsatellite data. Ecology and Evolution 4: 2450-2471. DOI: $\underline{10.1002 / \text { ece } 3.1108 .}$.

Villar-Salvador P. 2003. Importancia de la calidad de planta en los proyectos de revegetación. In Rey-Benayas JM, T Espigares-Pinilla, JM Nicolau-Ibarra eds. Restauración de Ecosistemas Mediterráneos. Alcalá de Henares, España. Universidad de Alcalá. p. 65-86.

Recibido: 30/03/20

Aceptado: 09/09/20 
